\title{
Fungus ball and emphysematous cystitis secondary to Candida tropicalis: A case report
}

\author{
Lei Wang, MD, PhD,; Xiang Ji, MD; Guo-feng Sun, MD;" Ying-chao Qin, MM;, Miao-zi Gong, MD;* \\ Jin-xia Zhang, MD; ${ }^{*}$ Ning-chen Li, MD,* Yan-qun Na, MD*
}

*Wu-Jieping Urology Center, Peking University Shougang Hospital, Peking University Health Science Center, Beiing, China; *Department of Pathology, Peking University Shougang Hospital, Peking University Health Science Center, Beiiing, China

Cite as: Can Urol Assoc J 2015;9(9-10):E683-6. http://dx.doi.org/10.5489/cuaj.3008 Published online September 9, 2015.

\section{Abstract}

Fungus ball and fungal emphysematous cystitis are two rare complications of fungal urinary tract infection. A 53-year-old male patient presented with these complications caused by Candida tropicalis simultaneously. The predisposing factors were diabetes mellitus and usage of broad-spectrum antibiotics. The fungus ball, measuring $3.5 \times 2.0 \mathrm{~cm}$ on the left wall of the urinary bladder, shrank significantly to $1.6 \times 0.8 \mathrm{~cm}$ after 5 days of intermittent irrigation with saline before surgery. With transurethral removal of the fungus ball and antifungal treatment with fluconazole, the patient fully recovered. We conclude that a bladder fungus ball and fungal emphysematous cystitis should always be suspected in patients with diabetes mellitus with uncontrolled funguria and abnormal imaging. Treatment should include a systemic antifungal therapy and thorough surgical removal of the fungus ball. A systemic antifungal therapy combined with a local irrigation with saline or antifungal drugs might help decrease the dissemination of fungemia during an invasive manipulation.

\section{Case report}

A 53-year-old male was referred to our department in February 2015 with intermittent, but recently aggravated cloudy urine. The symptom had been ongoing for 9 years, sometimes with frequency and urgency. No blood urine or fever was ever reported. To control the symptoms of cystitis, the patient underwent multiple treatments with broadspectrum antibiotics during this 9 -year period. Twice, fungus was found in his urine (9 years and 6 years ago).

The patient had uncontrolled type II diabetes mellitus for more than a decade. He also suffered from a right kidney stone and underwent a percutaneous nephrolithotomy 18 years ago. Physical examination was unremarkable. A routine urine test showed significantly elevated red blood cells (56.1/HP, normal $<4.5 / \mathrm{HP})$ and white blood cells $(328.7 / \mathrm{HP}$, normal $<5.4 / \mathrm{HP}$ ). Blood tests were normal, except for an elevated fasting glucose level of 7.7 (range: 3.6-6.1) $\mathrm{mmol} / \mathrm{L}$. Sonography revealed a medium- to hypo-echoic bladder tumour, measuring $3.5 \times 2.0 \mathrm{~cm}$. It was located on the left wall of the urinary bladder, did not move with posture changes, and had no sign of blood flow (Fig. 1a). A computed tomography $(\mathrm{CT})$ revealed no intravesical mass in either the unenhanced or dual-enhanced phase. Only a very faint filling defect was found in the excretory phase. Moreover, a large round gas collection was found in the bladder, and a $1.1 \times 0.9-\mathrm{cm}$ calculus revealed a right distal ureter, leading to mild unilateral hydronephrosis and ureterectasis (Fig. 1b).

Urinary cytology showed a large number of hyphae and neutrophil granulocytes. Candida tropicalis was isolated from the urine, but the blood culture was negative. Because a fungus ball secondary to Candida tropicalis was highly suspected at this time, intermittent bladder washouts with saline ( $2 \mathrm{~L}$ per time and 3 times per day) were performed via an indwelling three-cavity urinary catheter. A mass of floccules were flushed out during early irrigation. A rechecked sonography after 5 days of irrigation showed that the bladder tumour shrank significantly to $1.6 \times 0.8 \mathrm{~cm}$ (Fig. 2a).

After a single dose of intravenous fluconazole $(200 \mathrm{mg})$, cystoscopy and ureteroscopic lithotripsy were performed under general anesthesia to remove the remnants of the fungus ball and right ureteral stone. Cystoscopy revealed an unsmooth, congested and inflamed bladder mucosa. A pale, amorphous, immobile mass, measuring about $1.5 \times 1.0 \mathrm{~cm}$ was observed. The nearby mucosa was covered with sporadic fungal biofilms (Fig. 2b). The mass and the biofilms were thoroughly removed. The bladder mucosa on the base of the mass was also biopsied.

The postoperative recovery was uneventful. Fluconazole was given intravenously at a dose of $200 \mathrm{mg}$ daily for 3 days. Then, the dose was changed to $100 \mathrm{mg}$ daily of oral fluconazole. The urine culture was negative shortly after the surgery. Histologically, the fungus ball was composed by 


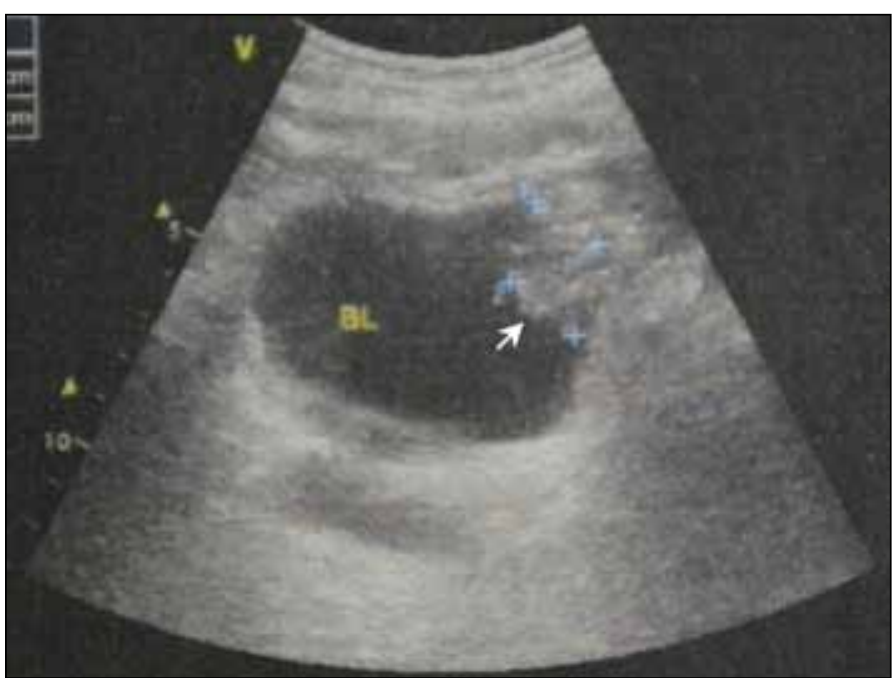

Fig. 1a. An ultrasound image illustrating a mass on the left wall of the urinary bladder (arrow), measuring $3.5 \times 2.0 \mathrm{~cm}$ with a medium- to hypo- echogenicity.

hyphae, spores, keratosic substances, and inflammatory exudate (Fig. 3a). The bladder mucosa on the base of the fungus ball exhibited chronic inflammation, squamous metaplasia and hyperkeratosis (Fig. 3b).

Following discharge, the patient received strict blood glucose control and oral fluconazole (100 mg, daily) for another 3 weeks. At the last follow-up, 2 months after the operation, a routine urine test was normal. The urine culture was also negative. Additionally, a CT scan showed no gas in the bladder.

\section{Discussion}

Fungus ball formation in the bladder is a rare complication of a fungal urinary tract infection. No more than 20 cases

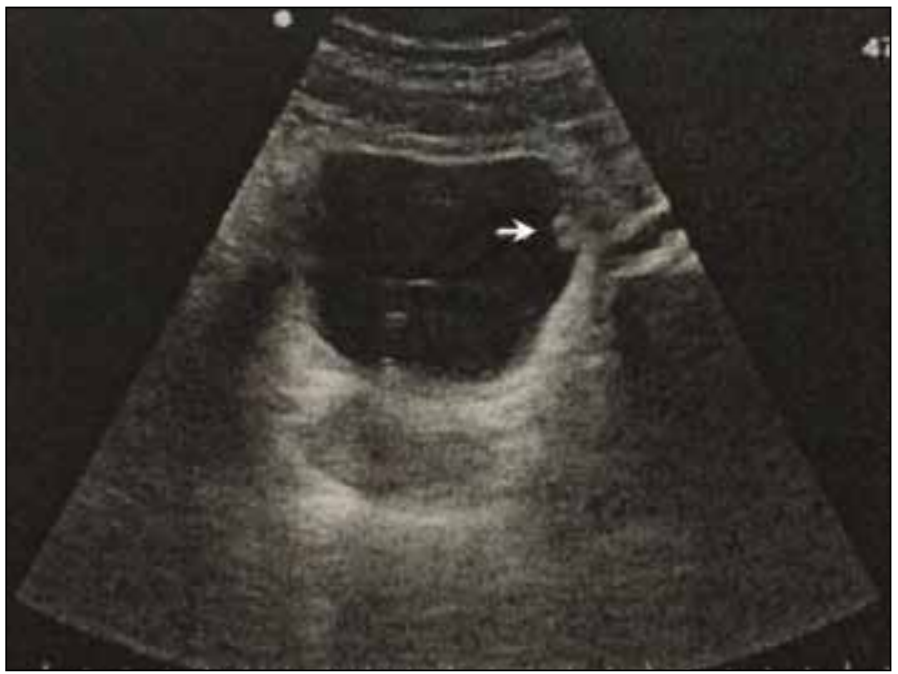

Fig. 2a. An ultrasound image revealed a significantly shrinkage of the bladder mass (arrow), measuring $1.6 \times 0.8 \mathrm{~cm}$, after intravesical irrigation with saline for a week.

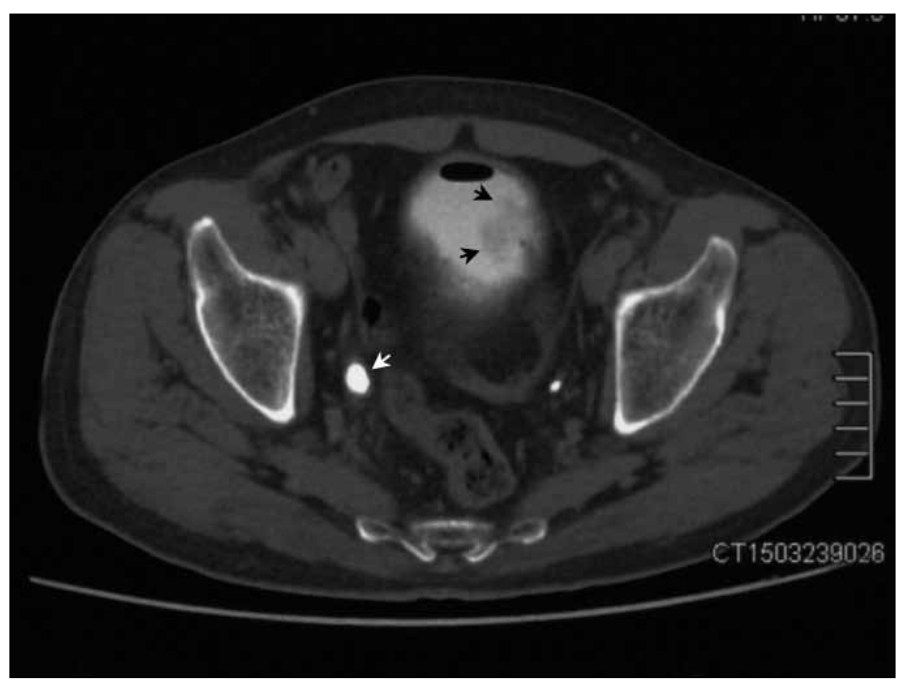

Fig. $1 \boldsymbol{b}$. Axial section of enhanced computed tomography (excretory phase) revealed round gas collections and a faintly filling-defect (black arrow) on the left wall of the urinary bladder. Right distal ureter was dialated (white arrow) due to the calculus lied beneath (not shown).

have been reported since it was first described in 1961. 1-8 Emphysematous cystitis, which almost exclusively occurs in patients with diabetes mellitus, is rare and is rarely the result of a fungus. ${ }^{9,10}$ To our knowledge, this is the second report of a bladder fungus ball occurring simultaneously with emphysematous cystitis. ${ }^{2}$ The predisposing factors were diabetes mellitus and usage of broad-spectrum antibiotics. An interesting and unique feature was that this fungus ball was loose and could be largely washed away with simply saline irrigation.

Fungus balls seldom occur in the urinary tract, but can lead to obstruction and hydronephrosis. ${ }^{11}$ Bladder fungus balls, also called fungus bezoars, ${ }^{2,7}$ are extremely rare and were described as an amorphous mass that could grow to as

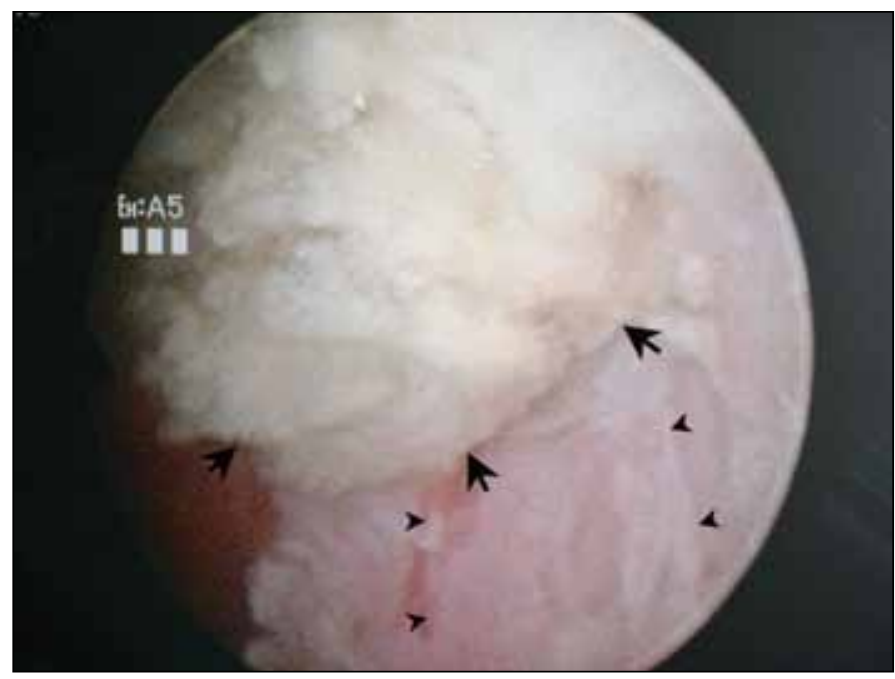

Fig. $\mathbf{2 b}$. An intraoperative image illustrating a pale, amorphous, immobile mass adhering to the left wall of the urinary bladder (arrows). The nearby mucosa was covered with sporadic fungal biofilms (arrowheads). 


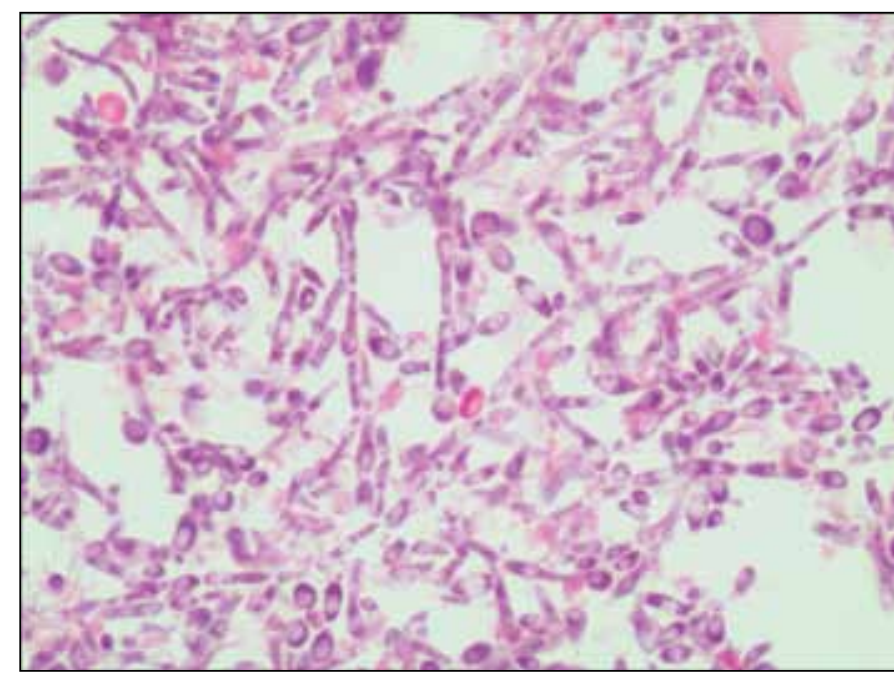

Fig. 3a. Histopathologic sections demonstrating the mass to be composed of Candida spores, hyphae, keratosic substances and inflammatory exudate.

large as $10 \mathrm{~cm}$ in size. ${ }^{3,4}$ It occurs exclusively in patients with risk factors and has an overall good prognosis with systemic antifungal agents and surgical removal. ${ }^{2,4,6}$ We suggest that a transurethral resection be the first surgical choice because it is minimally invasive. If the fungus ball is big and solid, open surgery should be considered. The most commonly used antifungal agents are fluconazole and amphotericin B deoxycholate because other drugs have extremely low urine concentrations.

For our patient, a major challenge was the proper management of the concurrent distal ureteral calculus, as mechanical trauma to the ureter during ureteroscopic lithotripsy might result in dissemination of Candida tropicalis into the bloodstream from the urine. We administered systemic fluconazole perioperatively and found no dissemination of a fungemia. As irrigation with an antifungal agent is still controversial, ${ }^{12}$ we irrigated the bladder with saline alone and got good results.

Emphysematous cystitis is characterized by gas collection within the bladder wall and lumen. This uncommon condition is usually observed in diabetic patients. Intramural and intraluminal gas arises from fermentation of excess glucose by a pathogen. Escherichia coli is the most common pathogenic micro-organism, while Candida spp. is very rare. ${ }^{2,9,10}$ Emphysematous cystitis is potentially life-threatening with a mortality rate of $7 \%$. However, early medical intervention can achieve a favourable prognosis. ${ }^{13}$

\section{Conclusion}

A bladder fungus ball and fungal emphysematous cystitis should always be suspected in patients with diabetes mellitus with uncontrolled funguria and abnormal imaging. The treatment should include a systemic antifungal therapy and thorough surgical removal of the fungus ball. A systemic

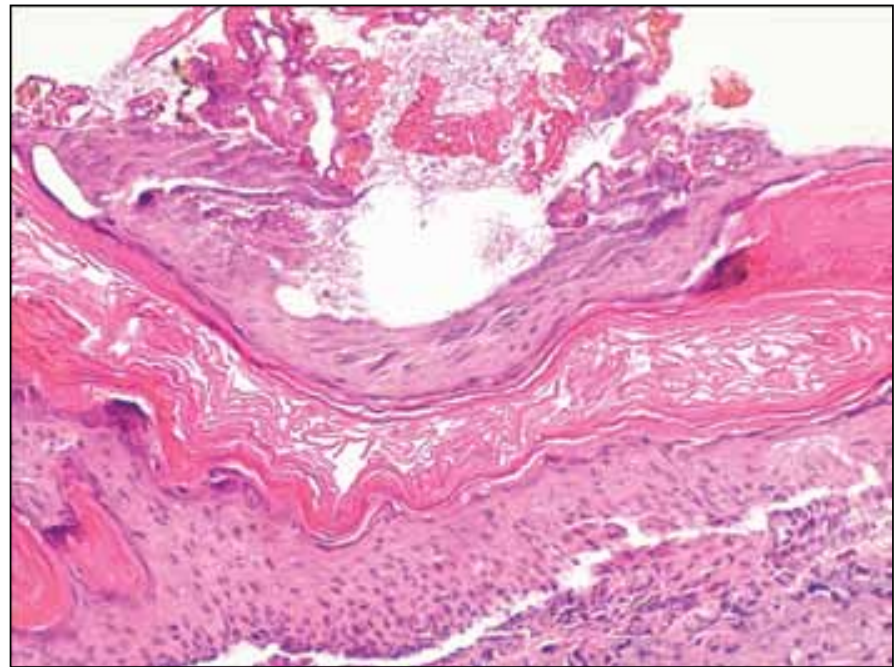

Fig. 3b.Bladder mucosa (from the base of the fungus ball) manifested chronic inflammation, squamous metaplasia and hyperkeratosis. There were no fungal elements within the bladder wall.

antifungal therapy combined with a local irrigation with saline or antifungal drugs might help to decrease the dissemination of fungemia during an invasive manipulation.

Acknowledgement: We wish to thank Carolyn D. who provided editing services on behalf of American Journal Experts.

Competing interests: The authors all declare no competing financial or personal interests.

This paper has been peer-reviewed.

\section{References}

1. Chisholm ER, Hutch JA. Fungus ball (Candida albicans) formation in the bladder. J Urol 1961;86:559-62.

2. Comiter CV, McDonald M, Minton J, et al. Fungal bezoar and bladder rupture secondary to candida tropicalis. Urology 1996;47:439-41. http://dx.doi.org/10.1016/S0090-4295(99)80470-1

3. Morton KM, Robertson AJ, Mclntyre J. Urinary bladder fungus ball. J Clin Pathol 1988;41:1243-4. httrp:// dx.doi.org/10.1136/icp.41.11.1243

4. González-Vicent M, Lassaletta A, López-Pino MA, et al. Aspergillus "fungus ball" of the bladder after hematopoietic transplantation in a pediatric patient: Successful treatment with intravesical voriconazole and surgery. Pediatr Transplant 2008;12:242-5. http://dx.doi.org/10.1111/j.1399-3046.2007.00871.x

5. Johnston 0 , Little DM, Hickey D, et al. Aspergillus "fungus ball" within a cadaveric renal transplant graft. Nephrol Dial Transplant 2004;19:1317-8. http://dx.doi.org/10.1093/ndt/ghh168

6. van Nieuwkoop C, Tijsterman JD, Van Dissel JT. Gather ye buds: Fungus formation of the bladder after complicated cesarean section. Am J Obstet Gynecol 2008;199:207.e1-2. http://dx.doi.org/10.1016/i. ajog.2008.06.001

7. Sundi D, Tseng K, Mullins JK, et al. Invasive fungal bezoar requiring partial cystectomy. Urology 2012;79:e21-22. http://dx.doi.org/10.1016/j.urology.2011.05.058

8. Krishnamurthy $R$, Aparaijtha C, Abraham $G$, et al. Renal aspergillosis giving rise to obstructive uropathy and recurrent anuric renal failure. Geriatr Nephrol Urol 1988;8:137-9. http://dx.doi. org/10.1023/A:1008343315440

9. Bartkowski DP, Lanesky JR. Emphysematous prostatitis and cystitis secondary to Candida albicans. J Urol 1988;139:1063-5. 
Wang et al.

10. Vaidyanathan $S$, Soni $B$, Hughes $P$, et al. Candida albicans fungaemia following traumatic urethral catheterisation in a paraplegic patient with diabetes mellitus and candiduria treated by caspofungin. Case Rep Infect Dis 2013;2013:693480. Epub 2013 0ct 8. http://dx.doi.org/10.1155/2013/693480

11. Shimada S, Nakagawa $H$, Shintaku I, et al. Acute renal failure as a result of bilateral ureteral obstruction by Candida albicans fungus balls. Int J Urol 2006;13:1121-2. http://dx.doi.org/10.1111/i.14422042.2006.01509.x

12. Drew RH, Arthur RR, Perfect JR. Is it time to abandon the use of Amphotericin B bladder irrigation? Clin Infect Dis 2005;40:1465-70. http://dx.doi.org/10.1086/429722
13. Amano M, Shimizu T. Emphysematous cystitis: A review of the literature. Intern Med 2014;53:79-82. http://dx.doi.org/10.2169/internalmedicine.53.1121

Correspondence: Dr. Ning-chen Li, Peking University Wu-Jieping, Urology Center, Peking University Shougang Hospital, 9* Jinyuanzhuang Road, Shiijingshan district, Beiijng, 100144, China; wipurologycenter@sina.com 